\title{
State-Of-The-Art in Microgrid-Integrated Distributed Energy Storage Sizing
}

\author{
Ibrahim Alsaidan ${ }^{1}$, Abdulaziz Alanazi ${ }^{1}$, Wenzhong Gao ${ }^{1}$, Hongyu $\mathrm{Wu}^{2}$ and Amin Khodaei ${ }^{1, *}$ \\ 1 Department of Electrical and Computer Engineering, University of Denver, Denver, CO 80210, USA; \\ Ibrahim.Alsaidan@du.edu (I.A.); Alanazi.Alanazi@du.edu (A.A.); Wenzhong.Gao@du.edu (W.G.) \\ 2 Department of Electrical and Computer Engineering, Kansas State University, Manhattan, KS 66506, USA; \\ HongyuWu@ksu.edu \\ * Correspondence: Amin.Khodaei@du.edu; Tel.: +1-303-871-2481
}

Received: 23 August 2017; Accepted: 12 September 2017; Published: 16 September 2017

\begin{abstract}
Distributed energy storage (DES) plays an important role in microgrid operation and control, as it can potentially improve local reliability and resilience, reduce operation cost, and mitigate challenges caused by high penetration renewable generation. However, to ensure an acceptable economic and technical performance, DES must be optimally sized and placed. This paper reviews the existing DES sizing methods for microgrid applications and presents a generic sizing method that enables microgrid planners to efficiently determine the optimal DES size, technology, and location. The proposed method takes into consideration the impact of DES operation on its lifetime to enhance the obtained results accuracy and practicality. The presented model can be used for both grid-tied (considering both grid-connected and islanded modes) and isolated microgrids.
\end{abstract}

Keywords: distributed energy storage; depth of discharge; microgrid expansion; mixed integer programming; optimal sizing

\section{Introduction}

The deployment of energy storage systems in distribution networks has considerably increased in recent years. Installed distributed energy storages (DESs) are owned by electric utilities or customers and used to provide a variety of services. For example, utilities deploy DESs to defer distribution network upgrades, improve reliability, or enhance voltage profile in their system. The customers on the other hand, install DESs to reduce their electricity payment by taking advantage of electricity price variations through an energy arbitrage or by reducing their potential demand charges. The attention toward DESs has also increased with the development of microgrids. According to the U.S. Department of Energy, a microgrid can be defined as "a group of interconnected loads and distributed energy resources (DERs) with clearly defined electrical boundaries that acts as a single controllable entity with respect to the grid and can connect and disconnect from the grid to enable it to operate in both grid-connected or islanded modes" [1].

There are two types of microgrids in terms of grid connection: grid-tied microgrids and isolated microgrids. In the first type, the microgrid and the main distribution network are connected via the point of common coupling (PCC). Grid-tied microgrids can disconnect themselves from the distribution network and operate in islanded mode, protecting their demand from external faults. The second microgrid type (i.e., isolated microgrid) is used to supply remote areas' demand for electricity where the connection to the utility grid is not available. Besides increasing the penetration of renewable distributed generators (DGs), microgrids provide other benefits to the electric utilities and end-users [2]. Such benefits include improving the system reliability, stability, and resiliency, reducing the power system losses, deferring system upgrades, and supplying electricity to rural areas among others. 
One of the essential components that facilitate the achievement of the aforementioned microgrid benefits is the DES. DES plays an important role in both isolated and grid-tied microgrids. In the isolated microgrid, DES is normally used to regulate the system frequency and voltage. The DES is either charged or discharged when a generation-demand mismatch occurs to maintain the stability of the microgrid voltage and frequency. In the grid-tied microgrid, the DES reduces the microgrid operation cost by efficiently utilizing the renewable generation and taking advantage of electricity price variations. In this case, the DES is charged during low electricity price periods or when the renewable DGs generation exceeds the summation of the local demands and the capacity of the line connecting the microgrid to the utility grid. The stored energy is then discharged at high price periods. Moreover, the DES can be used to smooth the transition between grid-connected and islanded modes of operation and to ensure a stable operation during islanding. Finally, for both microgrid types, the DES is used to improve the microgrid reliability and security by reducing the impact of unplanned incidents, such as generators or lines outages, on the microgrid operation.

The installation of DES into microgrids has been thoroughly investigated in literature. The research conducted in this area can be divided into two main topics: planning and operation. The primary objective of the DES planning is to determine the optimal DES size. Besides the optimal size, some of the existing works in literature investigate the optimal DES technology and location. Under operation research, the focus is towards proposing control and scheduling methods which are used to manage the DES operation in regard to a specific objective. However, the most important factor which determines the ability of the DES to deliver the desired economical and technical benefits is their size.

Different methods have been proposed in literature to size the DES for microgrid applications. In this paper, a comprehensive literature review of existing methods for DES sizing in microgrids is presented. It must be mentioned that this work focuses only on DES and hence excludes any work that considers the optimal sizing of other distributed energy resources in the planning problem. The existing DES sizing methods for both grid-tied and isolated microgrids are discussed and evaluated. In addition, a generic DES sizing method that can be used by microgrid planners to upgrade their system is proposed. Besides finding the optimal size, the proposed model can determine the optimal technology and location for the installed DES, taking into consideration the impact of DES operation on its lifetime.

\section{Microgrid-Integrated DES Sizing Methods}

The DES units are commonly sized based on their power ratings and energy ratings. The power rating is defined as the rate with which the DES can supply energy while the energy rating indicates the maximum amount of energy that can be delivered to the demand at each cycle [3]. Using these two parameters, the DES one-cycle charge/discharge duration can be determined. In this paper, the existing microgrid-integrated DES sizing methods are categorized based on their sizing objective into: (a) cost-based sizing methods and (b) non-cost-based sizing methods. In the cost-based sizing methods, the DES is sized to either minimize the total cost or maximize the total benefits associated with installing the DES within the microgrid. In the non-cost-based sizing methods, the DES is sized to provide technical services such as frequency control, voltage regulation, and power smoothing. In such methods, the economic aspect of the problem is ignored. The following subsections discuss each category in detail.

\subsection{Cost-Based DES Sizing Methods}

The investment cost associated with purchasing, installing, operating, and disposing of the DES is greatly related to their size. The installation of the DES is economically justifiable only if the provided economic benefits outweigh the investment cost. Most of the reviewed papers formulate the DES sizing problem as an optimization problem whose objective is either to minimize the total microgrid cost or to maximize the total benefits (i.e., a cost-benefit analysis). The DES size is considered as a design variable whose optimal value is determined by solving the optimization problem. Figure 1 shows the 
typical DES sizing cost components, which are divided into two categories: microgrid operation cost and DES investment cost. The former includes any operation cost needed to supply the microgrid local load such as the fuel cost and the cost of energy exchanged with the utility grid. It must be noted that the utility power exchange cost is only considered for grid-tied microgrids. Nevertheless, the reviewed papers may include all or some of the microgrid total cost components depicted in Figure 1.

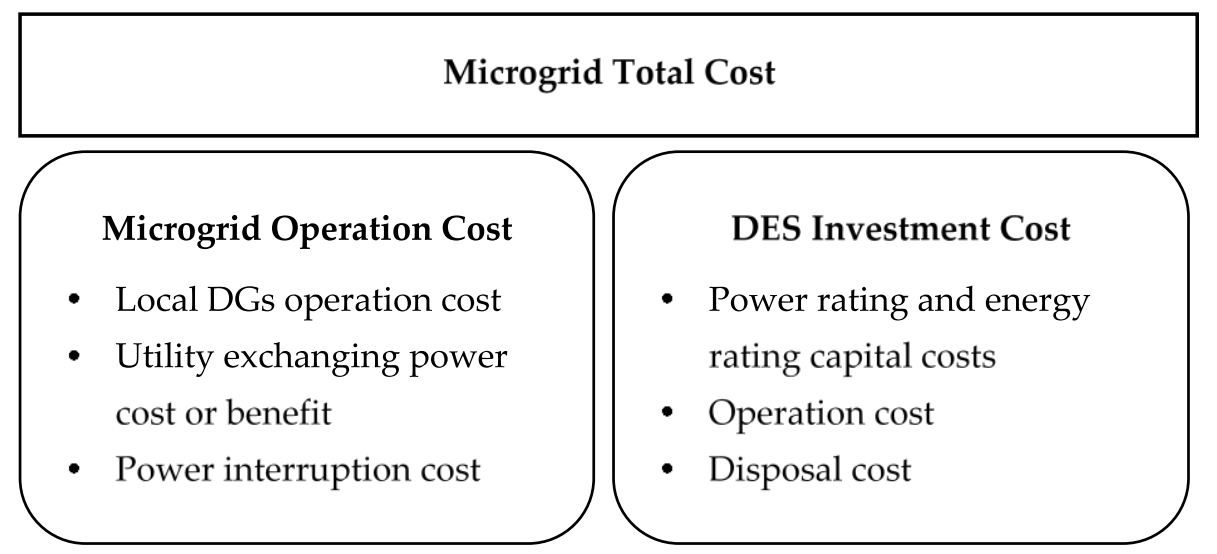

Figure 1. Microgrid-integrated distributed energy storage (DES) sizing cost components. DG: distributed generator.

The works in [4-10] implement mixed integer linear programming (MILP) to formulate the DES sizing problem. In [4], the renewable generation is not considered and the DES is sized for a microgrid containing only dispatchable DGs which reduces the potential economic benefits of the DES and ignores one of the most important aspects of microgrids. However, this work is expanded in [5] to consider not only renewable generation but also a reliability criterion. Different scenarios for the power system conditions such as generator outages and line contingencies as well as renewable generation are stochastically produced using Monte Carlo Simulation (MCS). After that, the large number of generated scenarios is reduced by a scenario reduction technique. A loss of load expectation (LOLE) index is used to evaluate the reliability of the studied microgrid. A DES capacity expansion model is developed in [6] for an isolated microgrid. In this work, the selected DES size is not considered fixed and is updated through the planning time horizon. It is found that the developed model reduces the associated cost by $10 \%$ as compared to fixed DES size methods. Similar to [5], this paper uses MCS to model the stochastic nature of wind speed, microgrid load, and DG availability, followed by a scenario reduction technique. The Ah-throughput is used as a measure for the DES lifetime, which is defined as the total amount of Ah or Wh that the DES is expected to deliver throughout the project lifetime before it needs to be replaced. The Ah-throughput is normally made readily available by the DES manufacturer. However, this method is not able to accurately determine the DES lifetime as the impact of important factors such as depth of discharge and number of cycles are overlooked. The work in [7] modifies the DES sizing problem formulation to determine not only the optimal size but also the optimal number of the installed DES units in the microgrid. A cap is imposed on the DES cycles performed each day in order to prolong the DES lifetime. It is shown that increasing the number of installed DES units increases the microgrid flexibility to take advantage of the electricity price variations. The work in [8] determines the optimal DES technology and size for an isolated microgrid. The DES degradation is considered in [9] and a model that finds the optimal depth of discharge along with the DES size is proposed. The work in [10] includes the installation year into the expansion problem and determines the optimal size and installation year for DES in an isolated microgrid that minimizes the total microgrid cost.

A genetic algorithm (GA) is used in [11] to develop the knowledge base for a fuzzy expert system that is used to manage the DES output power and solve a daily unit commitment problem 
in order to minimize the microgrid operation cost. In this work, the DES is sized using GA while its charging/discharging schedules are determined based on a fuzzy expert system. For economic reasons, the model proposed in [11] does not impose a minimum state of charge limit on the DES. Instead, a new cost associated with operating the DES at low state of charge is introduced to the objective function to prevent unnecessary deep discharge incidents. Similar to [6], the aging of the installed DES is modelled based on the weighted Ah-throughput. In this model, a weighting factor corresponding to the DES state of charge is multiplied by the amount of the actual Ah delivered to obtain what is called the effective cumulative Ah. This effective cumulative Ah is divided by the expected Ah that the DES is presumed to deliver when it is first installed to determine the DES loss of life. In [12], a hybrid GA-sequential quadratic programming (SQP) is used to optimize the size and the location of the DES units and capacitors in a smart grid. The SQP is used to solve the optimal power flow while the GA is used to determine the optimal size and location of the DES units and the capacitors that minimize the overall planning cost. A non-dominated sorting genetic algorithm II (NSGA-II) is employed in [13] to solve a multi-objective DES sizing problem in presence of demand response (DR). The considered objectives are to maximize the photovoltaic consumptive rate and the net profit of the microgrid. Reference [14] adopts a clustering technique to generate a number of scenarios associated to the wind speed, solar radiation, and load daily patterns to be used in DES sizing. GA is implemented to solve the proposed optimization problem as well.

The work in [15] studies DES sizing considering the stochastic nature of wind generation. A Here-and-Now approach is implemented to model the variability of wind generation by including new constraints to the microgrid unit commitment formulation. Particle swarm optimization (PSO) method is used to find the optimal DES size that maximize the microgrid total benefit in the grid-connected mode and minimize the microgrid total cost in the islanded mode. By decomposing the DES sizing problem into two subproblems (i.e., a planning subproblem and an energy management subproblem), the work in [16] develops a two-stage optimization strategy in order to reduce the computation time required to find the optimal DES size. An improved PSO is applied to solve the planning subproblem while the Mesh Adaptive Direct Search black box optimization algorithm is implemented to solve the microgrid energy management subproblem. The authors of $[17,18]$ study the optimal DES sizing in the presence of DR to regulate the frequency and voltage of a grid-tied microgrid during islanding. A multi-objective function is developed aiming to minimize the DES capital cost, maintenance and operating cost, as well as the size required to maintain the microgrid stability. A quantum-behaved particle swarm optimization (QSPO) is used in [19] to optimize the size of a hybrid energy storage system (HESS) that is composed of batteries and ultracapacitors. The authors compare the obtained results to the one obtained using conventional PSO and find that the QSPO is faster in solving the optimization problem.

A new evolutionary optimization algorithm is improved and adopted by the authors in [20] to determine the optimal energy rating of a DES installed in a grid-tied microgrid. The new algorithm is called Bat Algorithm (BA) and is described as a population-iterative based method. The proposed improved BA (IBA) results are compared to other optimization methods such as conventional BA, teaching-learning-based optimization (TLBO), and artificial bee colony (ABS) in terms of the resulted error from conducted test functions. In general, it is shown that the IBA yields smaller error values, in terms of best value, mean value, and standard deviation, compared to the other methods. Another new evolutionary optimization algorithm known as grey wolf optimization (GWO) is applied in [21] to solve the DES sizing problem in a microgrid. The obtained microgrid operation cost at the optimal DES size along with other optimization parameters such as standard deviation and simulation time are compared to those obtained by different optimization methods including the aforementioned IBA. GWO shows a superior performance compared to other optimization methods. The stochastic nature of the microgrid demand, renewable generation, and electricity price is considered in [22]. A scenario-based model is developed to formulate the unit commitment problem. The impact of the DES size on the microgrid operation cost is further investigated. 
An iterative based method is implemented in [23-26] to determine the optimal DES size. The microgrid unit commitment problem is solved for different DES sizes within predetermined minimum and maximum values, as shown in Figure 2. The unit commitment problem is solved by implementing dynamic programming (DP) in [23], knowledge based expert system controller (KBES) in [24], mixed integer nonlinear programming (MINLP) in [25], and MILP in [26]. The work in [23] focuses on determining the optimal power rating and energy rating of a Vanadium Redox Battery (VRB), taking into account the nonlinear relationship between the VRB power and efficiency. Different DES technologies are considered in [24] and it is found that the minimum energy cost is obtained when a lead acid battery is installed which makes it the optimal DES technology choice for the considered microgrid. The problem of reserve sizing and DES sizing is investigated in [27]. The authors propose a two-stage probabilistic co-optimization method that determines the optimal DES size as well as the reserve amount that minimizes the microgrid total cost with the consideration of the system reliability. The sizing problem is decomposed into a master problem in which the DES size is fixed and a subproblem in which the optimal reserve size is calculated. The DES size is then updated and the process is repeated. The optimal solution (i.e., the DES optimal size and the optimal reserve) would be the one that minimizes the microgrid total cost. In order to reduce the calculation time, a Markovian steady state analysis is implemented to solve the subproblem and find the optimal reserve value.

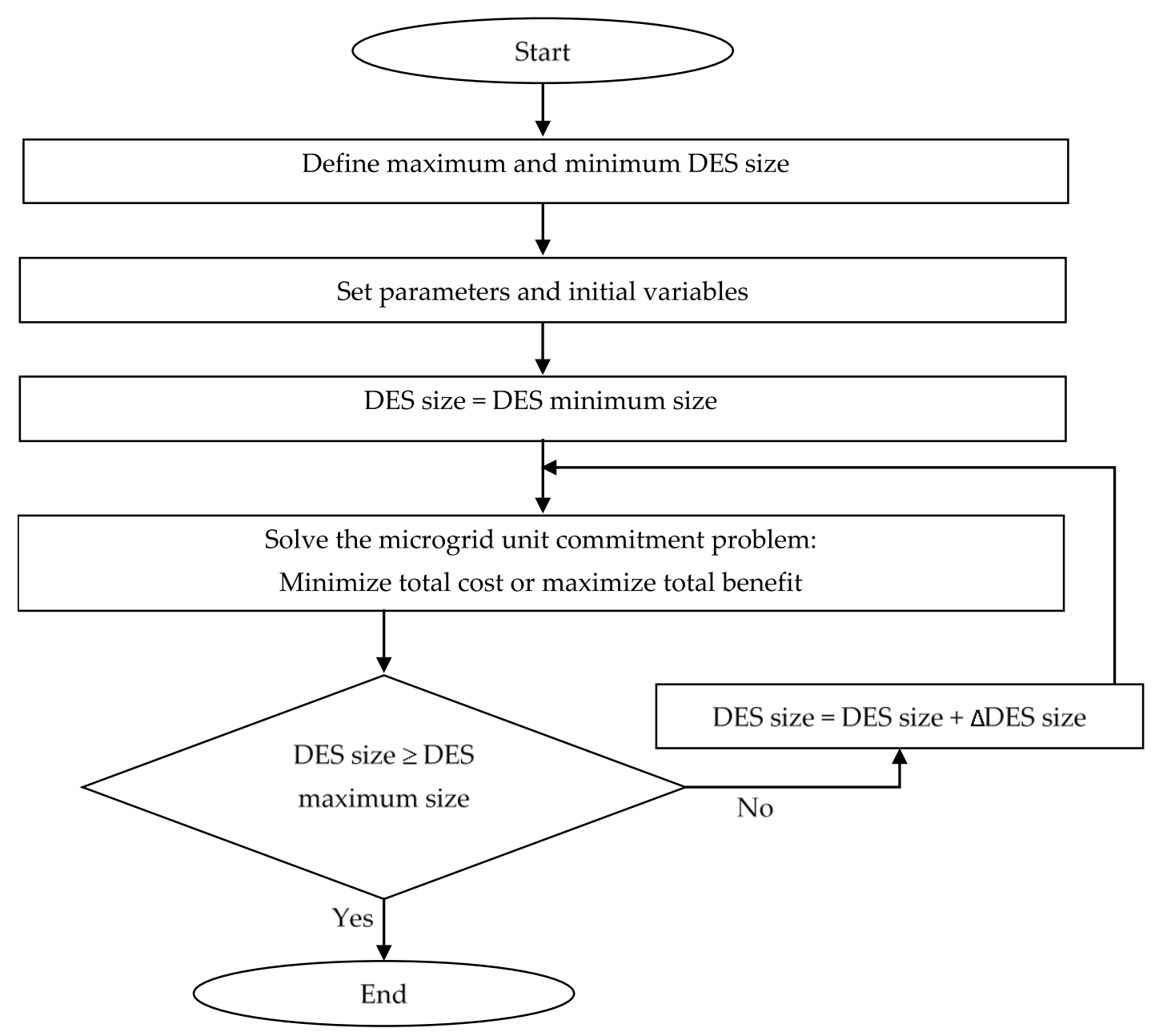

Figure 2. General flowchart for sizing DES using iterative-based methods. 


\subsection{Non-Cost-Based DES Sizing Methods}

The common aspect of the previous reviewed works is that they size the DES based on an economic objective. However, the following papers approach the DES sizing problem from a different perspective. A duty cycle-based sizing method is used in [28] in order to determine the size of a DES to be used for peak shaving applications. The DES cycling and the temperature impact on the sizing problem are considered and included as factors that adjust the determined size. However, it is not clear how the authors determine the values of these factors. In [29], the installed DES is analytically sized in order to smooth the power oscillation seen by the utility grid. A control algorithm is also developed to protect the DES from being over charged or discharged. The authors in [30] size the DES in order to minimize the power transferred through the line connecting the microgrid to the utility grid. The idea behind this is to reduce the dependency of the microgrid on the utility grid which will lead to improved microgrid reliability during islanded operation. The DES size and location are determined in [31] for both grid-connected and islanded microgrids simultaneously. A GA is used to solve the microgrid AC power flow. The fitness function is selected to minimize the power losses and improve the voltage profile. In [32], authors use a HESS which consists of batteries and supercapacitors to improve the power quality when integrating wind power in islanded microgrids. Supercapacitors can smooth the wind power with high frequency whereas the low frequency part of wind power is smoothed by batteries. The optimization problem is modeled by a Back Propagation neural network approach and solved in short term (to test the wind power smoothing) and long term (to prove the economic viability of the model).

The appropriate size for DES to regulate the frequency of an islanded microgrid is investigated in [33-37]. Reference [33] proposes a DES size optimization method based on an artificial neural network (ANN) model. The model inputs are the islanded microgrid frequency and voltage, and the output is the optimal DES size that is obtained after training the data using a multilayer perceptron structure. The multilayer perceptron structure can ensure high accuracy of data fitting so the error of obtained optimal sizing is very small. Moreover, the effect of the DES location has been investigated and it is found that the optimal location should be close to local loads to minimize power losses. In [34], the DES is optimized by analyzing the value of power ramp rate (PRR) of the microgrid. The case study shows the effect of the DES on the frequency control with and without considering the PRR. It is shown that the energy of DES that is essential for frequency control is remarkably reduced with the PRR consideration. The DES in [35] is used as a primary frequency controller to utilize the overloading characteristics of DES to restore the mismatch power during islanding transition in microgrids. The optimal DES capacity should be able to capture the maximum mismatch power. So, the mismatch power is calculated first to determine the DES overload capacity. The largest overloading charge or discharge power to restore the mismatch power is considered as the optimal power rating of the DES. An inertia based method is proposed in [36] to size the DES considering primary control (arrest the deviated frequency) and secondary control (restore the deviated frequency). The inertia deficiency for primary and secondary controls are measured as the key parameter of the DES sizing. The provided power from the DES may result in voltage violation, hence, the voltage stability is enhanced by using power electronics. A HESS is presented in [37] as an islanded microgrid frequency controller. The frequency is controlled based on hysteretic loop control to prolong battery lifetime by preventing small charge/discharge cycles, while a statistical model based on MCS is applied to determine the optimal capacity distributions of the HESS. The HESS output power is determined and analyzed through a simulation process on the system data. The optimal rated power of the battery is determined to depend on the maximum charging or discharging power in all cycles, while the optimal rated energy is the integration of all charging and discharging power in each single cycle. Similar to a battery, the supercapacitor distribution capacity is found.

From reviewing the existing DES sizing methods, the following can be concluded: 
- Some of the reviewed papers adopt a short planning horizon such as one day. In this case, the seasonal variations of the renewable generation, load demand, and electricity price are not considered in the DES sizing problem. As a result, the determined optimal size may not be accurate and the installed DES performance will be affected.

- The impact of the DES operating factors such as depth of discharge and number of cycles on its lifetime (i.e., DES degradation) are rarely included in the reviewed papers. This may result in the necessity to replace the DES before the end of the considered project lifetime which imposes an extra cost to the planning problem that has not been considered when the DES is sized.

- Most of the papers consider only one DES technology in the planning problem. Moreover, the optimal location at which the DES should be installed within the microgrid is normally ignored.

\section{Proposed DES Sizing Method}

In this section, a generic, cost-based, microgrid-integrated DES sizing model is proposed. The proposed model can be used for both grid-connected and islanded operation modes. The optimal DES technology, size, and location are selected to be the optimization variables. The solution of the optimization problem will return the optimal values for these variables along with the microgrid local units' schedules that minimize the microgrid total cost. The impact of DES operation on its lifetime is taken into consideration when the optimization problem is solved. The sizing problem is formulated as MILP. The objective function is expressed as:

$$
\operatorname{Min}\left(\sum_{e \in \mathrm{E}} I C_{e}+\sum_{t \in \mathrm{T}} O C_{t}\right)
$$

where IC and OC indicate the DES investment cost and the microgrid operation cost, respectively. The indices $e$ and $t$ represent the DES technology and the time interval. It must be noted that the DES investment cost is determined based on the DES size (i.e., power rating and energy rating) and technology. Different DES technologies possess different technical and economical characteristics, such as capital cost, roundtrip efficiency, depth of discharge versus number of cycle curve, among others. The proposed model enables the microgrid planners to consider these characteristics and to accordingly find the optimal technology that minimizes the microgrid total cost. Equation (1) is solved subject to a set of operational and physical constraints. These constraints include dispatchable and nondispatchable DGs constraints, power balance constraint, distribution network power flow and line capacity limits, and online reserve requirement, among others. For the sake of generalization, these constraints are left for the user to define as different microgrid planners may impose different operational requirements, however, details can be found in [38]. In this paper, the focus will be towards the DES operation modelling which shows how the impact of DES operation on its lifetime can be included in the DES sizing problem. Such impact is normally ignored in existing DES sizing methods, as discussed in the previous section.

The proposed model enables the microgrid planners to investigate the economic feasibility of DES installation in an existing microgrid. If the DES installation is economically feasible, the model will determine the DES optimal size, technology, location, and charging/discharging schedules. The model can be combined with any of the reviewed MIP-based methods in Section 2, i.e., [4-10], to improve the accuracy of the obtained results.

\subsection{Impact of DES Operation on Lifetime}

The two main operation factors that impact the DES degradation are depth of discharge and number of charging/discharging cycles. A curve that exhibits the DES lifecycle relation to its depth of discharge is normally provided by DESs manufacturers. An example of this curve is depicted in Figure 3. This relationship can be linearized using Piece wise linearization technique as shown in Figure 4 and used in DES sizing method to model DES degradation. 


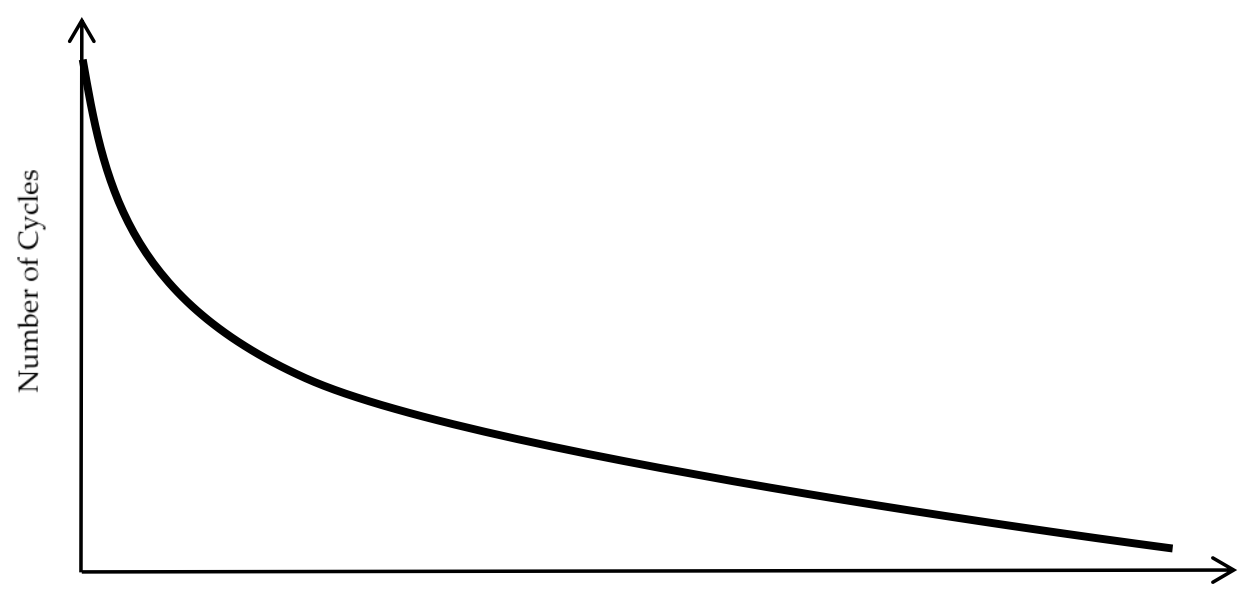

Depth of Discharge

Figure 3. An example of DES depth of discharge vs. number of cycles.

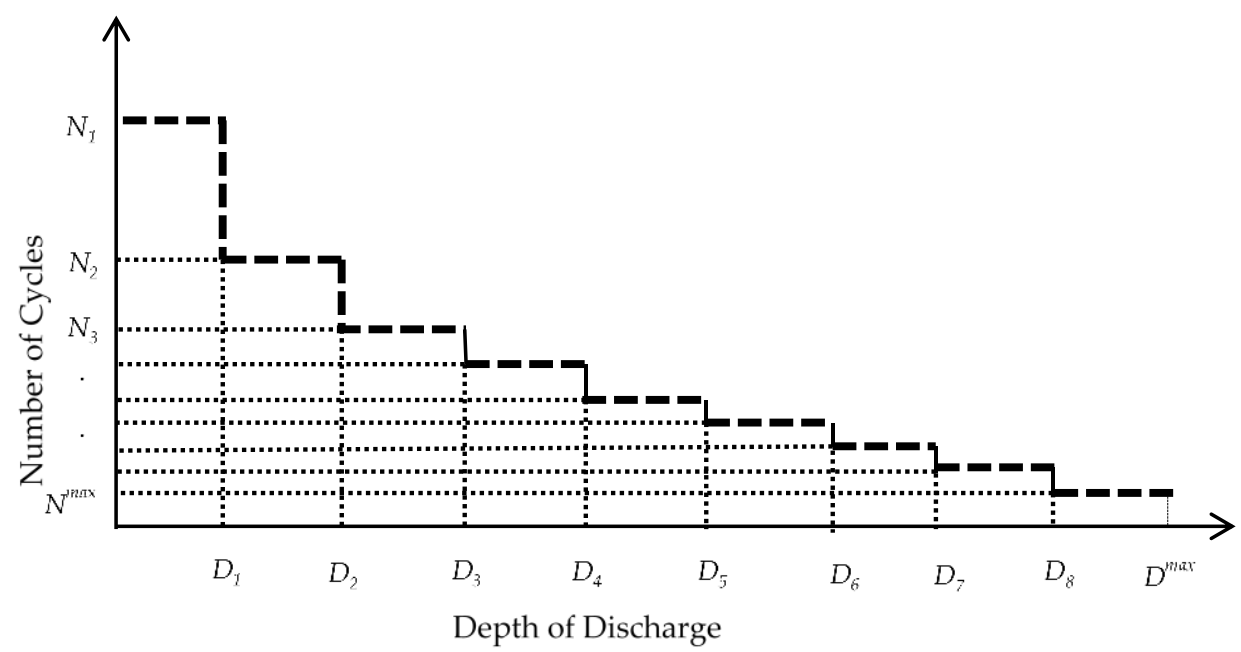

Figure 4. An example of linearized depth of discharge vs. number of cycles.

In this work, a novel parameter that represents the DES degradation is defined. This parameter can be derived from the linearized depth of discharge versus lifecycle curve using Equation (2).

$$
\alpha_{e m}=\frac{N^{\max }}{N_{m}} \quad \forall e \in \mathrm{E}, \forall m \in \mathrm{M}
$$

Thus, from Equation (2), the DES degradation parameter $(\alpha)$ value at each charging/discharging cycle depends on three factors: the DES technology, as different technology has different depth of discharge versus lifecycle curves, the number of cycles at maximum depth of discharge $\left(N^{\max }\right)$, and the number of cycles at the actual depth of discharge $\left(N_{m}\right)$. Once the actual depth of discharge at each performed charging/discharging cycle is calculated, the associated values of $N^{\max }$ and $N_{m}$ can be found from the linearized depth of discharge versus number of cycles curve given in Figure 4. That is, if the DES is discharged at its maximum depth of discharge value, the degradation parameter value will be 1 , otherwise it will be less than 1 . Figure 5 shows an example of the proposed novel DES degradation parameter. By this way, an accurate estimation of the DES lifetime based on depth of discharge and number of cycles can be included into the DES sizing problem, which will lead to improved accuracy and practicality of the obtained results. The mathematical equations used to model 
the DES operation as well as the impact of the DES operation on its lifetime are discussed in detail in the following sections.

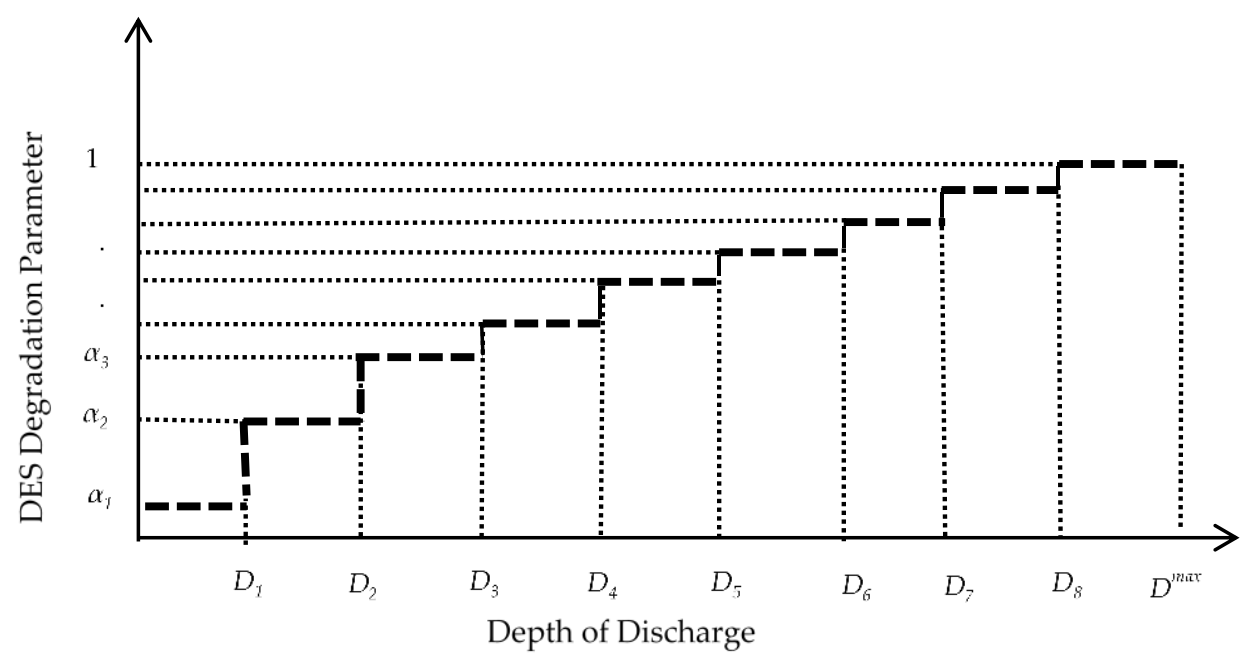

Figure 5. An example of DES degradation parameter.

\subsection{Proposed DES Sizing Mathematical Formulation}

The DES units are sized based on their power rating in $\mathrm{kW}\left(y_{e b}\right)$ and energy rating in $\mathrm{kWh}\left(x_{e b}\right)$. It must be noted that the DES are normally manufactured in a modular based size. Thus, the installed DES is formed by an integer multiple of the DES manufactured base modular size $\left(\Delta x_{e}\right)$ as in Equation (4). $k_{n}$ is an integer variable whose value starts from 0 and increases by 1 with each incremental step $n$, whereas $\gamma_{e b n}$ is a binary variable that indicates the optimal size, technology, and location of the installed DES. Thus, $\gamma_{e b n}$ will be 1 for the optimal DES technology $e$, bus number $b$, and energy rating size segment $n$, and 0 otherwise. In Equation (6), the DES power rating size is determined based on the desired discharging period $(\tau)$. The discharging period depends on the DES technology as some technologies are associated with a long discharging period (e.g., multiple hours) while other technologies have short discharging periods (e.g., multiple minutes).

$$
\begin{array}{ll}
x_{e}^{\min } \leq x_{e b} \leq x_{e}^{\max } & \forall e \in \mathrm{E}, \forall b \in \mathrm{B} \\
x_{e b}=\sum_{n} \gamma_{e b n}\left(x_{e}^{\min }+k_{n} \Delta x_{e}\right) & \forall e \in \mathrm{E}, \forall b \in \mathrm{B} \\
\sum_{n} \gamma_{e b n} \leq 1 & \forall e \in \mathrm{E}, \forall b \in \mathrm{B} \\
\frac{x_{e b}}{\tau_{e}^{\max }} \leq y_{e b} \leq \frac{x_{e b}}{\tau_{e}^{\min }} & \forall e \in \mathrm{E}, \forall b \in \mathrm{B}
\end{array}
$$

The DES operation (i.e., charging and discharging) can be modeled using Equations (7)-(11). The binary variable $u$ is used in Equations (7) and (8) to indicate the DES operation state; If $u$ is one, the DES is discharging and if $u$ is zero the DES is either charging or is at idle mode. By this, it is ensured that the DES does not charge and discharge at the same time. The operation state binary variable $u$ can be used to determine when the DES completes a charging/discharging cycle as in Equation (9). That is, the cycle indicator $\xi_{\text {ebt }}$ will be one every time a charging/discharging cycle is completed and 0 otherwise. The amount of energy stored in the DES at each time interval $\left(C_{e b t}\right)$ can be calculated using Equation (10). The DES round trip efficiency $(\eta)$ is considered during discharging process. The energy stored within the DES cannot exceed the determined optimal energy rating and cannot go below the 
determined minimum value which is computed based on the maximum depth of discharge as shown in Equation (11).

$$
\begin{array}{ll}
-y_{e b}\left(1-u_{e b t}\right) \leq P_{e b t}^{\mathrm{ch}} \leq 0 & \forall e \in \mathrm{E}, \forall b \in \mathrm{B}, \forall t \in \mathrm{T} \\
0 \leq P_{e b t}^{\mathrm{dch}} \leq y_{e b} u_{e b} & \forall e \in \mathrm{E}, \forall b \in \mathrm{B}, \forall t \in \mathrm{T} \\
\xi_{e b t}=\left(u_{e b t}-u_{e b(t-1)}\right) u_{e b t} & \forall e \in \mathrm{E}, \forall b \in \mathrm{B}, \forall t \in \mathrm{T} \\
C_{e b t}=C_{e b(t-1)}-\frac{P_{e b t}^{\mathrm{dch}}}{\eta_{i}}-P_{e b t}^{\mathrm{ch}} & \forall e \in \mathrm{E}, \forall b \in \mathrm{B}, \forall t \in \mathrm{T} \\
\left(1-D_{e}^{\mathrm{max}}\right) x_{e b} \leq C_{e b t} \leq x_{e b} & \forall e \in \mathrm{E}, \forall b \in \mathrm{B}, \forall t \in \mathrm{T}
\end{array}
$$

As discussed in the previous section, the DES operation has a great impact on its lifetime. This impact must be included in the DES sizing problem in order to enhance the obtained results' accuracy and practicality. Ignoring such impact may lead to the necessity of replacing the DES before the end of the project lifetime which imposes extra cost that is not considered at the planning stage. The DES degradation caused by operation factors is taken into consideration in the proposed sizing method by adopting the relationship between the DES depth of discharge and lifecycle. Equations (12)-(16) are used to accurately estimate the DES degradation over the planning horizon and to further ensure that the installed DES does not need to be replaced before the end of the considered project lifetime. The state of charge $\left(\phi_{e b t}\right)$ at each complete charging/discharging cycle is determined using Equation (12). Note that if a charging/discharging cycle is not completed (i.e., $\xi_{e b}=0$ ), the value of $\phi_{\text {ebt }}$ must be 1 which forces the depth of discharge value in Equation (13) to be 0 . The binary variable $z_{e b m t}$ is used to indicate the actual depth of discharge segment $(m)$ which will be used in Equation (15) to estimate the DES lifetime degradation. The DES degradation parameter $\alpha$ is calculated for each depth of discharge segment $m$ using Equation (2). The summation of the DES performed cycles multiplied by the DES estimated degradation parameter over the planning horizon must be less than the number of cycles that the DES can perform at the maximum depth of discharge, as in Equation (16).

$$
\begin{array}{cl}
\varphi_{e b t} x_{e b}=x_{e b}-C_{e b t} \xi_{\text {ebt }} & \forall e \in \mathrm{E}, \forall b \in \mathrm{B}, \forall t \in \mathrm{T} \\
\varphi_{e b t}=\sum_{m}\left(1-D_{e m}\right) z_{e b m t} & \forall e \in \mathrm{E}, \forall b \in \mathrm{B}, \forall m \in M, \forall t \in \mathrm{T} \\
\sum_{m} z_{e b m t} \leq 1 & \forall e \in \mathrm{E}, \forall \mathrm{b} \in \mathrm{B}, \forall t \in \mathrm{T} \\
\lambda_{e b t}=\sum_{m} z_{e b m t} \alpha_{e m} & \forall e \in \mathrm{E}, \forall \mathrm{b} \in \mathrm{B}, \forall t \in \mathrm{T} \\
\sum_{t} \lambda_{e b t} \xi_{e b t} \leq N^{\max } & \forall e \in \mathrm{E}, \forall \mathrm{b} \in \mathrm{B}
\end{array}
$$

To this end, the model outputs can be divided into two types: DES design variables and operation variables. The DES design variables are the optimal size, technology, and location which are denoted by $x_{e b}, y_{e b}$, and $\gamma_{e b n}$. These variables are determined for the entire planning horizon. The DES operation variables, on the other hand, are determined for each considered time interval $(t)$ within the planning horizon. These variables are the charging/discharging schedule $\left(u_{e b t}, C_{e b t}, P_{e b t}^{\mathrm{ch}}, P_{e b t}^{\mathrm{dch}}\right)$, the DES state of charge $\left(\phi_{e b t}\right)$, and the DES cycle indicator $\left(\xi_{e b t}\right)$. Along with these output variables, the model will determine the value for the auxiliary variables used to solve the problem $\left(\lambda_{e b t}, z_{e b m t}\right)$. The following parameters are provided as inputs to the model in order to get the optimal solution:

- Microgrid operational and physical constraints such as line capacity limits and bus voltage limits. These constraints are selected by the microgrid planner and added to the formulation. 
- Candidate DES technologies and characteristics such as power rating and energy rating capital costs, modular size $\left(\Delta x_{e}\right)$, roundtrip efficiency $\left(\eta_{e}\right)$, discharging periods $(\tau)$, and maximum depth of discharge $\left(D_{e}^{\max }\right)$.

- The manufacturer depth of discharge versus lifecycle curve for each candidate DES technology, which will be linearized and used to derive the DES degradation parameter $\left(\alpha_{\mathrm{em}}\right)$.

\subsection{The Proposed DES Sizing Model vs. Existing Models}

The reviewed DES sizing methods along with the presented method in this paper are summarized in Table 1 in terms of considered microgrid type, DES optimized characteristics, impact of operation factors in DES lifetime, and planning timeframe. A single day planning timeframe or less is labeled as short term, whereas a one-year planning timeframe or longer is labeled as long term. It is worth mentioning that the reviewed literature as well as the proposed model in this paper are intended to size a DES integrated into small to medium sized microgrids (i.e., in the range of hundreds of $\mathrm{kW}$ to single digit MW). It can be seen from Table 1 that most of the previous works focus on either grid-connected or islanded microgrid operation but not both. Moreover, none of the previous works in DES sizing in microgrids, except the works in $[6,9,11]$ in which a simple DES degradation model is proposed, consider the impact of the depth of discharge on the DES lifecycle and planning decisions. Besides this work, the various DES technologies' characteristics are considered only in $[8,24]$ while the optimal location is considered in [12,31]. Finally, it is important to mention that in order to improve the accuracy of the obtained optimization results, a long planning time horizon must be adopted. If a short timeframe, such as one day, is chosen, the determined solution (i.e., the optimal size) cannot guarantee a good performance since the seasonal variations in the demand and renewable generation are not taken into account.

Table 1. Summary of DES sizing methods for microgrid applications.

\begin{tabular}{|c|c|c|c|c|c|c|c|c|c|}
\hline \multirow{2}{*}{$\begin{array}{l}\text { Reference } \\
\text { Number }\end{array}$} & \multicolumn{2}{|c|}{ Microgrid Operation Mode } & \multicolumn{4}{|c|}{ DES Optimized Characteristics } & \multirow{2}{*}{$\begin{array}{c}\text { Impact of DES } \\
\text { Operation on Lifetime }\end{array}$} & \multicolumn{2}{|c|}{ Planning Timeframe } \\
\hline & Grid-Connected & $\begin{array}{l}\text { Isolated or } \\
\text { Islanded }\end{array}$ & $\begin{array}{l}\text { Power } \\
\text { Rating }\end{array}$ & $\begin{array}{l}\text { Energy } \\
\text { Rating }\end{array}$ & Technology & Location & & Short & Long \\
\hline $\begin{array}{c}1-10 \\
{[4]}\end{array}$ & $\sqrt{ }$ & 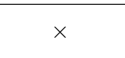 & $\sqrt{ }$ & $\times$ & $\times$ & $\times$ & $\times$ & $\times$ & $\sqrt{ }$ \\
\hline [5] & $\sqrt{ }$ & $\times$ & $\sqrt{ }$ & $\sqrt{ }$ & $\times$ & $\times$ & $\times$ & $\times$ & $\sqrt{ }$ \\
\hline [6] & $\times$ & $\sqrt{ }$ & $\sqrt{ }$ & $x$ & $\times$ & $\times$ & $\sqrt{ }$ & $\times$ & $\sqrt{ }$ \\
\hline [7] & $\sqrt{ }$ & $x$ & $\sqrt{ }$ & $\sqrt{ }$ & $x$ & $x$ & $\times$ & $x$ & $\sqrt{ }$ \\
\hline [8] & $\times$ & $\sqrt{ }$ & $\sqrt{ }$ & $\sqrt{ }$ & $\sqrt{ }$ & $x$ & $x$ & $\times$ & $\sqrt{ }$ \\
\hline [11] & $\sqrt{ }$ & $x$ & $\sqrt{ }$ & $\sqrt{ }$ & $\times$ & $\times$ & $\sqrt{ }$ & $\sqrt{ }$ & $\times$ \\
\hline [12] & $\sqrt{ }$ & $x$ & $\sqrt{ }$ & $\times$ & $\times$ & $\sqrt{ }$ & $\times$ & $\times$ & $\sqrt{ }$ \\
\hline [13] & $\sqrt{ }$ & $x$ & $x$ & $\sqrt{ }$ & $\times$ & $\times$ & $x$ & $\times$ & $\sqrt{ }$ \\
\hline [14] & $\times$ & $\sqrt{ }$ & $\times$ & $\sqrt{ }$ & $\times$ & $\times$ & $\times$ & $\sqrt{ }$ & $\times$ \\
\hline [15] & $\sqrt{ }$ & $\sqrt{ }$ & $\times$ & $\sqrt{ }$ & $\times$ & $\times$ & $\times$ & $\sqrt{ }$ & $\times$ \\
\hline [16] & $\sqrt{ }$ & $\sqrt{ }$ & $\sqrt{ }$ & $\sqrt{ }$ & $x$ & $x$ & $\times$ & $x$ & $\sqrt{ }$ \\
\hline [22] & $\sqrt{ }$ & $\times$ & $\times$ & $\sqrt{ }$ & $\times$ & $\times$ & $\times$ & $\sqrt{ }$ & $\times$ \\
\hline [23] & $\sqrt{ }$ & $\sqrt{ }$ & $\sqrt{ }$ & $\sqrt{ }$ & $\times$ & $\times$ & $\times$ & $\sqrt{ }$ & $\times$ \\
\hline [24] & $\times$ & $\sqrt{ }$ & $\sqrt{ }$ & $\sqrt{ }$ & $\sqrt{ }$ & $\times$ & $\times$ & $\times$ & $\sqrt{ }$ \\
\hline [25] & $x$ & $\sqrt{ }$ & $\times$ & $\sqrt{ }$ & $x$ & $x$ & $x$ & $\sqrt{ }$ & $\times$ \\
\hline [26] & $\sqrt{ }$ & $\sqrt{ }$ & $x$ & $\sqrt{ }$ & $x$ & $x$ & $x$ & $\sqrt{ }$ & $x$ \\
\hline [27] & $\sqrt{ }$ & $\sqrt{ }$ & $\times$ & $\sqrt{ }$ & $x$ & $x$ & $\times$ & $\times$ & $\sqrt{ }$ \\
\hline [28] & $\times$ & $\sqrt{ }$ & $x$ & $\sqrt{ }$ & $x$ & $\times$ & $\times$ & $\sqrt{ }$ & $x$ \\
\hline [29] & $\sqrt{ }$ & $\times$ & $\sqrt{ }$ & $\sqrt{ }$ & $\times$ & $\times$ & $\times$ & $\sqrt{ }$ & $\times$ \\
\hline [30] & $\sqrt{ }$ & $x$ & $\sqrt{ }$ & $\times$ & $\times$ & $x$ & $\times$ & $\sqrt{ }$ & $\times$ \\
\hline [31] & $\sqrt{ }$ & $\sqrt{ }$ & $\sqrt{ }$ & $\times$ & $\times$ & $\sqrt{ }$ & $\times$ & $\sqrt{ }$ & $\times$ \\
\hline [32] & $x$ & $\sqrt{ }$ & $x$ & $\sqrt{ }$ & $x$ & $\times$ & $x$ & $\sqrt{ }$ & $x$ \\
\hline [33] & $\times$ & $\sqrt{ }$ & $\sqrt{ }$ & $x$ & $\times$ & $\times$ & $\times$ & $\sqrt{ }$ & $\times$ \\
\hline [34] & $\times$ & $\sqrt{ }$ & $\sqrt{ }$ & $\sqrt{ }$ & $\times$ & $\times$ & $\times$ & $\sqrt{ }$ & $\times$ \\
\hline
\end{tabular}




\section{Conclusions}

In this paper, a comprehensive literature review of the existing microgrid-integrated DES sizing methods was provided. The reviewed methods were divided based on their sizing objective into two main categories of cost-based and non-cost-based sizing methods. The former included the methods that size the DES in order to minimize or maximize an economic objective. The latter, however, included the methods that size the DES in order to perform a desired technical service such as frequency regulation, voltage regulation, or power smoothing. In addition, a generic DES sizing model was proposed and discussed which can be potentially converted to any of the proposed cost-based models with proper simplifications. The proposed model can be used by microgrid planners to upgrade their microgrids with DES. Besides the installed DES optimal size, the model could determine the optimal technology, location, and charging/discharging schedule while taking into consideration the impact of DES operation on its lifetime.

Author Contributions: Ibrahim Alsaidan and Abdulaziz Alanazi performed the literature survey and wrote the main parts of the paper. Wenzhong Gao and Amin Khodaei advised and helped the two first authors to propose the new models and develop the associated formulation. Hongyu $\mathrm{Wu}$, as a subject matter expert, reviewed the entire work to ensure practicality and contributions to the field.

Conflicts of Interest: The authors declare no conflict of interest. The founding sponsors had no role in the design of the study; in the collection, analyses, or interpretation of data; in the writing of the manuscript, and in the decision to publish the results.

\section{References}

1. Department of Energy Office of Electricity Delivery and Energy Reliability. Summary Report: 2012 DOE Microgrid Workshop. 2012. Available online: http://energy.gov/sites/prod/files/2012\%20Microgrid\% 20Workshop\%20Report\%2009102012.pdf (accessed on 1 November 2015).

2. Parhizi, S.; Lotfi, H.; Khodaei, A.; Bahramirad, S. State of the Art in Research on Microgrids: A Review. IEEE Access 2015, 3, 890-925. [CrossRef]

3. Eyer, J.; Corey, G. Energy Storage for the Electricity Grid: Benefits and Market Potential Assessment Guide; Sandia National Laboratories: Albuquerque, NM, USA, 2010; Volume 20, p. 5.

4. Bahramirad, S.; Daneshi, H. Optimal sizing of smart grid storage management system in a microgrid. In Proceedings of the IEEE PES Innovative Smart Grid Technologies (ISGT), Washington, DC, USA, 16-20 January 2012; pp. 1-7.

5. Bahramirad, S.; Reder, W.; Khodaei, A. Reliability-Constrained Optimal Sizing of Energy Storage System in a Microgrid. IEEE Trans. Smart Grid 2012, 3, 2056-2062. [CrossRef]

6. Hajipour, E.; Bozorg, M.; Fotuhi-Firuzabad, M. Stochastic Capacity Expansion Planning of Remote Microgrids With Wind Farms and Energy Storage. IEEE Trans. Sustain. Energy 2015, 6, 491-498. [CrossRef]

7. Alsaidan, I.; Khodaei, A.; Gao, W. Distributed energy storage sizing for microgrid applications. In Proceedings of the IEEE/PES Transmission and Distribution Conference and Exposition (T\&D), Dallas, TX, USA, 3-5 May 2016; pp. 1-5.

8. Alsaidan, I.; Khodaei, A.; Gao, W. Determination of battery energy storage technology and size for standalone microgrids. In Proceedings of the IEEE Power and Energy Society General Meeting (PESGM), Boston, MA, USA, 17-21 July 2016; pp. 1-5.

9. Alsaidan, I.; Khodaei, A.; Gao, W. Determination of optimal size and depth of discharge for battery energy storage in standalone microgrids. In Proceedings of the North American Power Symposium (NAPS), Denver, CO, USA, 18-20 September 2016; pp. 1-6.

10. Alharbi, H.; Bhattacharya, K. Optimal Sizing of Battery Energy Storage Systems for Microgrids. In Proceedings of the IEEE Electrical Power and Energy Conference (EPEC), Calgary, AB, Canada, 12-14 November 2014; pp. 275-280.

11. Fossati, J.P.; Galarza, A.; Martín-Villate, A.; Fontán, L. A method for optimal sizing energy storage systems for microgrids. Renew. Energy 2015, 77, 539-549. [CrossRef]

12. Carpinelli, G.; Celli, G.; Mocci, S.; Mottola, F.; Pilo, F.; Proto, D. Optimal Integration of Distributed Energy Storage Devices in Smart Grids. IEEE Trans. Smart Grid 2013, 4, 985-995. [CrossRef] 
13. Zhou, N.; Liu, N.; Zhang, J.; Lei, J. Multi-Objective Optimal Sizing for Battery Storage of PV-Based Microgrid with Demand Response. Energies 2016, 9, 591. [CrossRef]

14. Jiao, B.; Wang, C.; Guo, L. Scenario Generation for Energy Storage System Design in Stand-alone Microgrids. Energy Procedia. 2014, 61, 824-828. [CrossRef]

15. Khorramdel, H.; Aghaei, J.; Khorramdel, B.; Siano, P. Optimal Battery Sizing in Microgrids Using Probabilistic Unit Commitment. IEEE Trans. Ind. Inform. 2016, 12, 834-843. [CrossRef]

16. Xiao, H.; Pei, W.; Yang, Y.; Kong, L. Sizing of battery energy storage for micro-grid considering optimal operation management. In Proceedings of the International Conference on Power System Technology (POWERCON), Chengdu, China, 20-22 October 2014; pp. 3162-3169.

17. Kerdphol, T.; Qudaih, Y.; Mitani, Y. Optimum battery energy storage system using PSO considering dynamic demand response for microgrids. Int. J. Electr. Power Energy Syst. 2016, 83, 58-66. [CrossRef]

18. Kerdphol, T.; Qudaih, Y.; Mitani, Y. Battery energy storage system size optimization in microgrid using particle swarm optimization. In Proceedings of the IEEE PES Innovative Smart Grid Technologies Conference Europe (ISGT-Europe), Istanbul, Turkey, 12-15 October 2014; pp. 1-6.

19. Xie, X.; Wang, H.; Tian, S.; Liu, Y. Optimal capacity configuration of hybrid energy storage for an isolated microgrid based on QPSO algorithm. In Proceedings of the 5th International Conference on Electric Utility Deregulation and Restructuring and Power Technologies (DRPT), Changsha, China, 26-29 November 2015; pp. 2094-2099.

20. Bahmani-Firouzi, B.; Azizipanah-Abarghooee, R. Optimal sizing of battery energy storage for micro-grid operation management using a new improved bat algorithm. Int. J. Electr. Power Energy Syst. 2014, 56, 42-54. [CrossRef]

21. Sharma, S.; Bhattacharjee, S.; Bhattacharya, A. Grey wolf optimisation for optimal sizing of battery energy storage device to minimise operation cost of microgrid. IET Gener. Transm. Distrib. 2016, 10, $625-637$. [CrossRef]

22. Mohammadi, S.; Mohammadi, A. Stochastic scenario-based model and investigating size of battery energy storage and thermal energy storage for micro-grid. Int. J. Electr. Power Energy Syst. 2014, 61, 531-546. [CrossRef]

23. Nguyen, T.A.; Crow, M.L.; Elmore, A.C. Optimal Sizing of a Vanadium Redox Battery System for Microgrid Systems. IEEE Trans. Sustain. Energy 2015, 6, 729-737. [CrossRef]

24. Ross, M.; Hidalgo, R.; Abbey, C.; Joós, G. Analysis of Energy Storage sizing and technologies. In Proceedings of the IEEE Electric Power and Energy Conference (EPEC), Halifax, NS, Canada, 25-27 August 2010; pp. 1-6.

25. Chen, S.X.; Gooi, H.B. Sizing of energy storage system for microgrids. In Proceedings of the IEEE 11th International Conference on Probabilistic Methods Applied to Power Systems (PMAPS), Singapore, 14-17 June 2010; pp. 6-11.

26. Chen, S.X.; Gooi, H.B.; Wang, M.Q. Sizing of Energy Storage for Microgrids. IEEE Trans. Smart Grid 2012, 3 , 142-151. [CrossRef]

27. Dong, J.; Gao, F.; Guan, X.; Zhai, Q.; Wu, J. Storage-Reserve Sizing With Qualified Reliability for Connected High Renewable Penetration Micro-Grid. IEEE Trans. Sustain. Energy 2016, 7, 732-743. [CrossRef]

28. Khasawneh, H.J.; Mondal, A.; Illindala, M.S.; Schenkman, B.L.; Borneo, D.R. Evaluation and sizing of energy storage systems for microgrids. In Proceedings of the IEEE/IAS 51st Industrial Commercial Power Systems Technical Conference (I\&CPS), Calgary, AB, Canada, 5-8 May 2015; pp. 1-8.

29. Koohi-Kamali, S.; Rahim, N.A.; Mokhlis, H. New algorithms to size and protect battery energy storage plant in smart microgrid considering intermittency in load and generation. In Proceedings of the 3rd IET International Conference on Clean Energy and Technology (CEAT), Kuching, Malaysia, 24-26 November 2014; pp. 1-7.

30. Keskamol, K.; Hoonchareon, N. Sizing of battery energy storage system for sustainable energy in a remote area. In Proceedings of the 2015 IEEE Innovative Smart Grid Technologies-Asia (ISGT ASIA), Bangkok, Thailand, 3-6 November 2015; pp. 1-4.

31. Beiranvand, A.; Aghdam, M.M.; Li, L.; Zhu, S.; Zheng, J. Finding the optimal place and size of an energy storage system for the daily operation of microgrids considering both operation modes simultaneously. In Proceedings of the 2016 IEEE International Conference on Power System Technology (POWERCON), Wollongong, NSW, Australia, 28 September-1 October 2016; pp. 1-6. 
32. Sun, C.; Yuan, Y. Sizing of hybrid energy storage system in independent microgrid based on BP neural network. In Proceedings of the 2nd IET Renewable Power Generation Conference (RPG 2013), Beijing, China, 9-11 September 2013; pp. 1-4.

33. Kerdphol, T.; Tripathi, R.N.; Hanamoto, T.; Khairudin; Qudaih, Y.; Mitani, Y. ANN based optimized battery energy storage system size and loss analysis for distributed energy storage location in PV-microgrid. In Proceedings of the 2015 IEEE Innovative Smart Grid Technologies-Asia (ISGT ASIA), Bangkok, Thailand, 3-6 November 2015; pp. 1-6.

34. Hongesombut, K.; Piroon, T.; Weerakamaeng, Y. Evaluation of battery energy storage system for frequency control in microgrid system. In Proceedings of the 2013 10th International Conference on Electrical Engineering/Electronics, Computer, Telecommunications and Information Technology (ECTI-CON), Krabi, Thailand, 15-17 May 2013; pp. 1-4.

35. Aghamohammadi, M.R.; Abdolahinia, H. A new approach for optimal sizing of battery energy storage system for primary frequency control of islanded Microgrid. Int. J. Electr. Power Energy Syst. 2014, 54, 325-333. [CrossRef]

36. Toliyat, A.; Kwasinski, A. Energy storage sizing for effective primary and secondary control of low-inertia microgrids. In Proceedings of the 2015 IEEE 6th International Symposium on Power Electronics for Distributed Generation Systems (PEDG), Aachen, Germany, 22-25 June 2015; pp. 1-7.

37. Jia, H.; Mu, Y.; Qi, Y. A statistical model to determine the capacity of battery-supercapacitor hybrid energy storage system in autonomous microgrid. Int. J. Electr. Power Energy Syst. 2014, 54, 516-524. [CrossRef]

38. Khodaei, A. Resiliency-Oriented Microgrid Optimal Scheduling. IEEE Trans. Smart Grid 2014, 5, $1584-1591$. [CrossRef]

(C) 2017 by the authors. Licensee MDPI, Basel, Switzerland. This article is an open access article distributed under the terms and conditions of the Creative Commons Attribution (CC BY) license (http:/ / creativecommons.org/licenses/by/4.0/). 